\title{
STABILITY ANALYSIS AND NUMERICAL SOLUTIONS OF A COMPETITION MODEL WITH THE EFFECTS OF DISTRIBUTION PARAMETERS
}

\author{
MD. KAMRUJJAMAN*, SADIA AKTER LIMA, SONIA AKTER AND TANZILA EVA \\ Department of Mathematics, University of Dhaka, Dhaka-1000, Bangladesh
}

\begin{abstract}
A system of two nonlinear differential equations in mathematical biology is considered. These models are originally stimulated by population models in biology when solutions are required to be non-negative, but the ordinary differential equations can be understood outside of this conventional scope of population models. The focus of this paper is on the use of linearization techniques, and Hartman Grobman theory to analyze nonlinear differential equations. We provide stability analysis and numerical solutions for these models that describe behaviors of solutions based only on the parameters used in the formulation of the systems.
\end{abstract}

Keywords: Stability analysis, Nonlinear system, Lyapunov functional, eigenvalue and eigenvector.

\section{INTRODUCTION}

Frequently, Mathematical models of real world affairs are formulated in terms of systems of nonlinear differential equations, which is not easy to solve explicitly. To defeat this obstacle, a qualitative way to analysis of solutions to nonlinear systems by making phase portraits and using stability analysis is adopted. These techniques in the analysis of a system of two nonlinear differential equations in mathematical biology are illustrated. The fact is that, allowing solutions for these equations to be positive provides some very attractive mathematical problems, and demonstrates the utility of the analysis techniques to be described in this work. The focus of this study is to discuss about the stability of system of nonlinear equations using linearization techniques (Morgan 2015), Hartman-Grobman theorem (Freedfman 1980, Kapur 1989, Perko 2008) and Lyapunov theorem (Perko 2008, Strogatz 2000, Wiggins 1983).

Usually given any possibly nonlinear system of first order ordinary differential equations, a qualitative characterization of the behavior of solutions is generated which depends on the given initial conditions. For doing this, at first equilibrium points are found and then using the nature of equilibrium points, the stability of the system of nonlinear ordinary differential equations (Wiggins 1983) can be checked. Runge-Kutta-Fehlberg method (Burden and Faires 1993) is also used for solving a system of non-linear differential equations. The focus of the work needs to be obtained quickly since there are some basic ideas which will not discuss in details. In preliminaries, differential equations are introduced which are classified as partial differential equation (Kapur 1989, Murray 1989, Wiggins 1983). Mainly in the following section, the analysis of stability will be discussed. For stability examination, the concept of equilibrium point which is a vector that satisfies $f(t, x(t))=0$ need to be considered.

Formally, an equilibrium point $x_{e}$ is stable if for $\left\|x\left(t_{0}\right)-x_{e}\right\|<\delta$; where $\delta$ is a positive scalar such that $\left\|x\left(t ; t_{0}, x_{0}\right)-x_{e}\right\|<\in$ for all $t \geq t_{0}$. When the equilibrium point $x_{e}$ is stable and convergent then it is known as asymptotically

*Corresponding author. Email: 〈kamrujjaman@du.ac.bd>. 
stable. Also the point $x_{e}$ is locally asymptotically stable (Taylor 1958) if $\left\|x(0)-x_{e}\right\|<r \Rightarrow \lim _{t \rightarrow \infty} x(t)=x_{e}$ and it is known as globally asymptotically stable (Taylor 1958) if it is asymptotically stable for all $x(0) \in \mathfrak{R}^{n}$. To apply this methodology, various types of growth functions are considered with chaos or crowding tolerance (Gilpin and Ayala 1973, Gompertz 1825, Smith 1963, Verhulst 1838).

The rest of the part of the paper is organized as follows. In sections 2 and 3, few examples have been established by solving the equilibriums and the equilibrium points are obtained. Among these equilibria, a number of them give stable solution and rest others are unstable solution. It is noted that the equilibrium points with negative real parts indicate the stable solution. Otherwise, the solutions are unstable.

In sections 3.1, 3.2 and 4.1, several well established theorems are discussed which are connected to the study of stability. After that a well recognized theorem known as Lasalle's Principle (LaSalle 1976) is recalled. The statement of Lasalle's theorem helps to execute global asymptotic stability of a system.

The main novelty of this paper is described shortly as follows:

- The result is established by using eigenvalue and eigenvector that implies the stability test of the model.

- In parametric case, it is considered that the intrinsic growth rate is constant as well as time varying.

- The similar type of study is introduced for the choice of carrying capacities which covered all type of possible variation.

- The main results of Theorems are verified theoretically as well as numerically.

- Finally, the study presents some robust theories of Lyapunov stability and Lasalle's principle and left for the readers for further study of the considered model.

\section{Preliminaries}

In the current section, the nonlinear system is introduced, and the linearization techniques of the system and various types of stability are considered.

\subsection{System of Nonlinear Differential Equations}

Example 1: An example of a system of nonlinear differential equations is the system of Lotka-Volterra equations (Murray 1989) in biology for two organisms such as

$$
\begin{aligned}
& \frac{d u}{d t}=(1-5 v) u \\
& \frac{d v}{d t}=(-1+5 u) v
\end{aligned}
$$

This is one of the sample competition models in mathematical biology that shows how the ecosystems are in balance between two species; may be the predator-prey. The main goal of current work is to discuss about the stability of the system of non-linear ordinary differential equation with logistic type non-symmetric growths. The next theorem (Freedfman 1980, Kapur 1989, Perko 2008) is important for further analysis.

\subsection{Hartman-Grobman Theorem}

A system involving in time with state $u(t) \in \mathfrak{R}^{n}$ that satisfies the differential equation $\frac{d u}{d t}=f(u)$ for some smooth map $f: \mathfrak{R}^{n} \rightarrow \mathfrak{R}^{n}$ is considered. It is supposed that the map has a hyperbolic equilibrium state $u^{*} \in \mathfrak{R}^{n}$; that is $f\left(u^{*}\right)=0$ and the Jacobian matrix $A=\left[\frac{\partial f_{i}}{\partial x_{j}}\right]$ of $f$ at state $u^{*}$ has no eigenvalue with real part equal to zero. Then there exists a neighborhood $N$ of the equilibrium $u^{*}$ and a homeomorphism $h: N \rightarrow \mathfrak{R}^{n}$ such that in the 
neighborhood $N$ the flow of $\frac{d u}{d t}=f(u)$ is topologically conjugate by the continuous map $U=h(u)$ to the flow of its linearization $\frac{d u}{d t}=A U$

Even for infinitely differentiable maps $f$, the homeomorphism $h$ need not be smooth, not even locally Lipschitz. However, it turns out to be Hölder continuous (Taylor 1958), with an exponent depending on the constant of hyperbolicityof $A$.

The Hartman-Grobman theorem has been extended to infinite dimensional Banach spaces, non-autonomous systems $\frac{d u}{d t}=f(u, t)$ and to cater for the topological differences that occur when there are eigenvalues with zero or nonzero real part.

Example 2: The following problem will show the techniques of linearization (Morgan 2015). It is supposed that the $2 D$ system in variables $w=(y, z)$ evolving according to the pair of coupled differential equation

$$
\begin{aligned}
& \frac{d y}{d t}=-3 y+y z \\
& \frac{d z}{d t}=z+y^{2}
\end{aligned}
$$

By direct computation it can be seen that the only equilibrium of this system lies at the origin, that is $w^{*}=0$ the coordinate transform, $w=h^{-1}(W)$ where $W=(Y, Z)$, given by

$$
\begin{aligned}
& y \approx Y+Y Z+\frac{1}{w^{2}} Y^{3}+\frac{1}{2} Y Z^{2} \\
& z \approx Z-\frac{1}{7} Y^{2}-\frac{1}{3} Y^{2} Z
\end{aligned}
$$

is a smooth map between the original $w=(y, z)$ and new $W=(Y, Z)$ coordinates, at least near the equilibrium at the origin. In the new coordinates the dynamical system transforms to its linearization form

$$
\frac{d y}{d t}=-3 y \text { and } \frac{d z}{d t}=z
$$

Hence the original dynamics in some finite neighborhood is obtained by a distorted version of the linearization.

\section{Stability Analysis of Nonlinear Systems}

The main purpose of this section is to understand the term stable means resistant to change. From this section, it will be possible for readers to find the stable points and unstable points for a system of nonlinear differential equations by using the equilibrium points of that system. This portion also includes that when a system of nonlinear differential equations is very critical and creates difficulties to find equilibrium points then linearization method can be used.

\subsection{Finding the Stability and Instability of} Equilibrium States

A system of nonlinear equations

$$
\left\{\begin{array}{l}
\frac{d u}{d t}=u(1-u-2 v) \\
\frac{d v}{d t}=v(1-2 u-v)
\end{array}\right.
$$

is first considered. The first aim is to find out the fixed (equilibrium) points. For this purpose set

$$
\begin{aligned}
& P=u(1-u-2 v)=0 \\
& Q=v(1-2 u-v)=0
\end{aligned}
$$

which yields the equilibrium points

$$
\left(u_{0}, v_{0}\right)=\{(0,0),(0,1),(1,0)\} .
$$

In this part, next approach is to find out the fourth fixed point while both populations are coexisting. For this case, the following two equations

$$
\begin{aligned}
& u+2 v=1 \\
& 2 u+v=1
\end{aligned}
$$

are considered. Finally, the equilibria 
$\left(u_{0}, v_{0}\right)=\left\{(0,0),(0,1),(1,0),\left(\frac{1}{3}, \frac{1}{3}\right)\right\}$

are obtained. The jacobian matrix be defined as:

$$
J_{(u, v)}=\left(\begin{array}{cc}
P_{u} & P_{v} \\
Q_{u} & Q_{v}
\end{array}\right)
$$

where, $P=u(1-u-2 v)$ and $\quad Q=v(1-2 u-v)$. Now differentiation of both $P$ and $Q$ with respect to $u$ and $v$ respectively and the Jacobian matrix is

$J_{(u, v)}=\left(\begin{array}{cc}1-2 v-2 u & -2 u \\ -2 v & 1-2 u-2 v\end{array}\right)$

Case 1: For the fixed point $(0,0)$

$$
J_{(0,0)}=\left(\begin{array}{ll}
1 & 0 \\
0 & 1
\end{array}\right)
$$

In this case the eigenvalues are 1 and 1 , which are real and positive. Then the trivial equilibrium $(0,0)$ is always unstable.

Case 2: For the fixed point $(1,0)$

$$
J_{(0,1)}=\left(\begin{array}{cc}
-1 & 0 \\
-2 & -1
\end{array}\right)
$$

In the second case, this is a lower triangular matrix and the eigenvalues are -1 and -1 , whose real components are negative and according to Hartman-Grobman Theorem the fixed point $(0,1)$ is stable.

Case 3: For the fixed point $(1,0)$

$$
J_{(1,0)}=\left(\begin{array}{cc}
-1 & -2 \\
0 & -1
\end{array}\right)
$$

In the third case, this is an upper triangular matrix. Hence, the eigenvalues are -1 and -1 , which are negative and real. Thus, the fixed point $(1,0)$ is stable.
Case 4: For the fixed point $\left(\frac{1}{3}, \frac{1}{3}\right)$

$$
J_{\left(\frac{1}{3}, \frac{1}{3}\right)}=\left(\begin{array}{rr}
-\frac{1}{3} & -\frac{2}{3} \\
-\frac{2}{3} & -\frac{1}{3}
\end{array}\right)
$$

Here,

$$
\begin{aligned}
& \operatorname{det}(J-\lambda I)=0 \\
& \Rightarrow \lambda^{2}+\frac{2}{3} \lambda-\frac{1}{3}=0 \\
& \Rightarrow \lambda=\left(-\frac{2}{3} \pm \sqrt{\frac{4}{9}+\frac{12}{9}}\right) / 2
\end{aligned}
$$

It is seen that one of them is positive and the left one is negative i.e. have opposite signs.

Therefore, the fixed point $\left(\frac{1}{3}, \frac{1}{3}\right)$ is unstable.

In the following example, generalized example to analyze the stability for another law of growth function is considered.

\subsection{Linearization Method to Check the Stability and Instability}

For this, the system of nonlinear equations with symmetric logistic type growths is considered and defined as follows

$$
\left\{\begin{array}{l}
\frac{d u}{d t}=u(k-u-v) \\
\frac{d v}{d t}=v(k-u-v)
\end{array}\right.
$$

And for simplicity for further analysis, $f(u, v)=u(k-u-v)$ and $\quad g(u, v)=v(k-u-v)$ are denoted. Where $u, v$ are the densities of the population of two specific species considered in the model and $k$ is the carrying capacity or environmental support and $u, v>0, k>0$.

The equilibria of the system is to be found and then the system is linearized as follows:

To find the fixed points, $f(u, v)=0, g(u, v)=0$ is considered; that is,

$$
\begin{aligned}
& u(k-u-v)=0 \\
& v(k-u-v)=0
\end{aligned}
$$


By solving, four fixed points:

$(0,0),(0, k),(k, 0),(\alpha k,(1-\alpha) k)$ are obtained.

Here, $(0,0)$ is the trivial solution, $(0, k),(k, 0)$

are semi-trivial solutions and $(\alpha k,(1-\alpha) k)$ is

the coexisting solution for all $\alpha \in(0,1)$.

For steady state analysis first the system (7)-(8) is linearized. The Jacobian matrix of $f$ and $g$ at each fixed point have to be found:

$$
J=\left(\begin{array}{ll}
f_{u} & f_{v} \\
g_{u} & g_{v}
\end{array}\right)
$$

Putting the values of $f_{u}, f_{u}, g_{u}$ and $g_{v}$ above

$$
J=\left(\begin{array}{cc}
k-2 u-v & -u \\
-v & k-u-2 v
\end{array}\right)
$$

is obtained. At the initial stage, the point at $(0,0)$ is considered and the Jacobian matrix is

$$
J_{(0,0)}=\left(\begin{array}{ll}
k & 0 \\
0 & k
\end{array}\right)
$$

such that the eigenvalues are $\lambda=k, k$. Since real parts of the eigenvalues are positive so the equilibrium point $(0,0)$ is unstable.

In the following step, the equilibirum point $(0, k)$ with Jacobian matrix is explored

$$
J_{(0, k)}=\left(\begin{array}{cc}
0 & 0 \\
-k & -k
\end{array}\right)
$$

Two eigenvalues, $\lambda=0,-k$ are obtained. In this issue the real parts of the eigenvalues are not positive, so the point $(0, k)$ is asymptotically stable. Now, at $(k, 0)$ the Jacobian matrix $J_{(k, 0)}=\left(\begin{array}{cc}-k & -k \\ 0 & 0\end{array}\right)$.

Hence, the eigenvalues are $\lambda=0,-k$. Since real parts of the eigenvalues are not positive as the previous one, so the point $(k, 0)$ is also asymptotically stable.
Now at the coexisting equilibrium $(\alpha k,(1-\alpha) k)$ the Jacobian matrix is

$$
J=\left(\begin{array}{cc}
-\alpha k & -\alpha k \\
(\alpha-1) k & (\alpha-1) k
\end{array}\right)
$$

which produce the eigenvalues $\lambda=0,-k$. Again non-positive real parts of the eigenvalues are obtained. So, the point $(\alpha k,(1-\alpha) k)$ is asymptotically stable.

Finally, introducing the intrinsic growth rates, the physical significance of the logistic model by numerical simulations is shown and the results are described in the following section.

\section{Competition Model with Logistic Growth}

The nonlinear differential equations (6) is recalled by introducing intrinsic growth rates with crowding effects

$$
\left\{\begin{array}{l}
\frac{d u}{d t}=r_{1} u\left(1-\frac{u+v}{k_{1}}\right) \\
\frac{d v}{d t}=r_{2} v\left(1-\frac{u+v}{k_{2}}\right)
\end{array}\right.
$$

Where $u(0)>0$ and $v(0)>0$ and $u, v$ are population densities of two species. The specific growth rates are $r_{1}, r_{2}$ with corresponding resource distributions $k_{1}, k_{2}$ respectively for respective populations.

\subsection{Main Results}

In this section, the following main results are established for the system (9) depending on the variations of parametric values. In the first result, the interest is to consider the constant growth rates while the carrying capacities vary.

Theorem 1: If $r_{1}=r_{2}=$ const then for any nonnegative and non-trivial initial values $u_{0}>0, v_{0}>0$, the following results are valid:

(i) For $k_{1}=k_{2}=k(t) \neq$ const, the coexistence solution is stable periodically. 
(ii) For $\quad k_{1}(t)>k_{2}(t)$, the semi-trivial equilibrium $\left(k_{1}(t), 0\right)$ is periodically globally asymptotically stable.

(iii) For $k_{1}(t)<k_{2}(t)$, the semi-trivial equilibrium $\left(0, k_{2}(t)\right)$ is periodically globally asymptotically stable.

The results in Theorem 1 are verified in Examples 4.1, 4.2 and 4.3 by particular functions of specific growth rates of organisms and resource distributions. One can check that the results are valid for functions connected to the real life problems when two species are in competition for natural resources. In the following results, it is shown that the growth rates are non-constant whereas the resource functions are constant.

Theorem 2: If $r_{1}=r_{2}=r(t) \neq$ const, then for any non-negative and non-trivial initial values $u_{0}>0, v_{0}>0$, the following results are valid:

(i) For $k_{1}=k_{2}=$ const, the coexistence solution is stable.

(ii) For $k_{1}>k_{2}$, the semi-trivial equilibrium $\left(k_{1}, 0\right)$ is globally asymptotically stable.

(iii) For $k_{1}<k_{2}$, the semi-trivial equilibrium $\left(0, k_{2}\right)$ is globally asymptotically stable.

The results of Theorem- 2 are thus justified in Examples 4.4, 4.7 and 4.8.
Next results show the effects of intrinsic growth rates:

Theorem 3: If $k_{1}=k_{2}=$ const, $r_{1} \neq$ const and $r_{2} \neq$ const then for any non-negative and nontrivial initial values $u_{0}>0, v_{0}>0$, the following results are valid:

(i) For $r_{1}(t)>>r_{2}(t)$, the coexistence solution $\left(u^{*}, v^{*}\right)$ is globally asymptotically stable.

(ii) For $r_{1}(t)<<r_{2}(t)$, the coexistence solution $\left(u^{*}, v^{*}\right)$ is globally asymptotically stable.

The results of Theorem- 3 are thus checked in Examples 4.5 and 4.6; for more details Example 4.10 can be seen for constant growth functions.

It is noted that all these results are possible to proof by considering the Jacobean matrix via Hartman-Grobman Theorem (Freedfman 1980, Kapur 1989, Perko 2008) or Lasalle's principle (LaSalle 1976) as shown in the previous Sections 3.1 and 3.2. Instead in this portion the results are verified using numerical examples and illustrations in the following Section 4.2.

Example 4.1. Identical intrinsic growth rates and equal carrying capacity are considered.
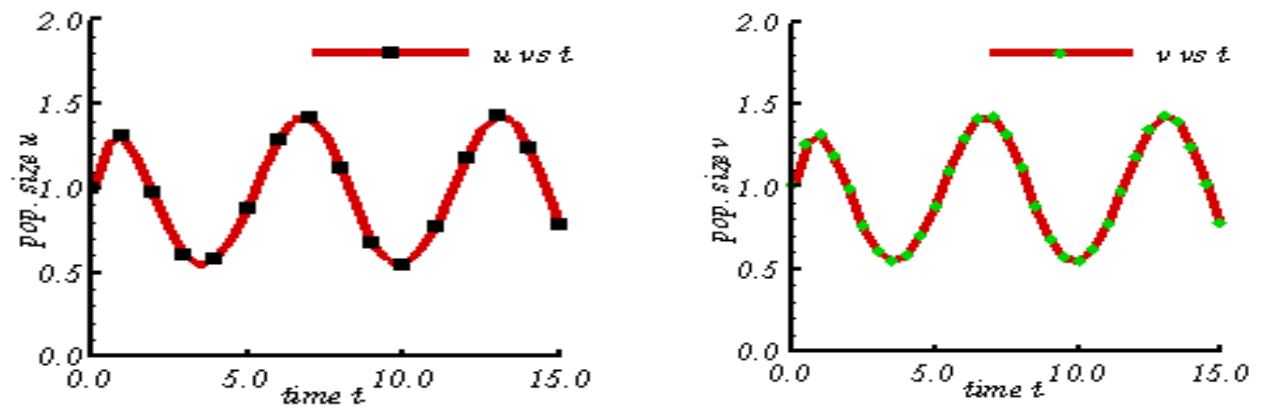

Fig. 1.1. Solutions of system (9) with respect to time for $r_{1}=r_{2}=2$ and $k_{1}=k_{2}=2+\cos (t)$ and for initial values $u_{0}=v_{0}=1$. 
Example 4.2. In this case, equal intrinsic growth rates and time dependent carrying capacities for $k_{2}>k_{1}$ are considered.
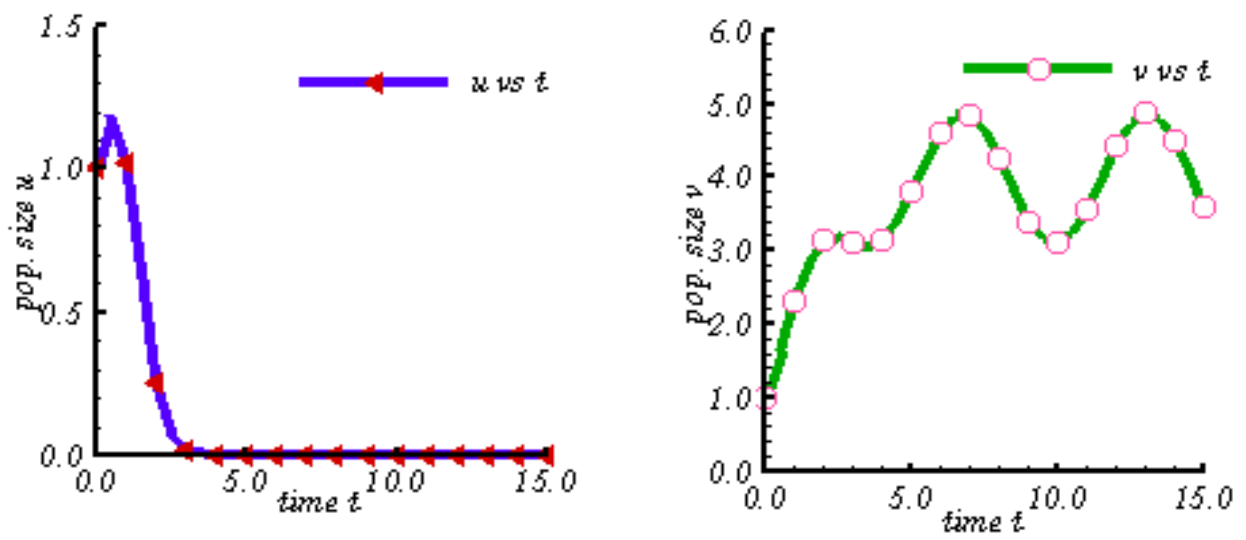

Fig. 1.2. Solutions of (9) with respect to time for $r_{1}=r_{2}=2$ and $k_{1}=2+\cos (t), k_{2}=4+\cos (t)$ and for initial values $u_{0}=v_{0}=1$.

\subsection{Numerical Examples}

The Runge-Kutta-Fehlberg method (Burden and Faires 1993) is introduced to solve the problem (9) simultaneously. The method also describes the required solutions graphically which assist us to understand the behaviors of solutions. Here, some examples to investigate the model are considered by showing the effect of different parameters. Different solutions for different values of those parameters are obtained.

The introductory example 4.1 presents constant and equal $r_{1}, r_{2}$ i.e. $r_{1}=r_{2}=2$ and $k_{1}=k_{2}=2+\cos (t)$ where $k_{1}, k_{2}$ are functions of time $t$ then two types of solutions for two population sizes are obtained which are represented graphically in Fig. 1.1. In Fig. 1.1, it is observed that both population sizes are coexisting for same initial densities and their densities are increasing and decreasing periodically.

In this example 4.2, it is noticed that the values of $r_{1}, r_{2}$ are constant and identical i.e. $r_{1}=r_{2}=2$ and $k_{1}=2+\cos (t), k_{2}=4+\cos (t)$ here $k_{1}, k_{2}$ are functions of time $t$ and $k_{1}<k_{2}$. The solutions of the model are represented graphically in Fig. 1.2. The left diagram of the Fig. 1.2, with the increase of time the densities of the population are increasing for short moment and then turning the density in decreasing order strictly and at last the population size tends to zero. The right design of the same figure depicts that with the increase of time the density of the population is increasing and sustains periodically.

Another example 4.3 where $r_{1}, r_{2}$ are equivalent and consider $r_{1}=r_{2}=2$ which shows that both of them are constant and $k_{1}=4+\cos (t)$, $k_{2}=2+\cos (t)$ where $k_{1}, k_{2}$ are functions of time $t$ and $k_{1}>k_{2}$ then two types of solutions are obtained for two population sizes which are presented graphically in Fig. 1.3 for identical initial densities. The left design of Fig. 1.3 indicates that with the increase of time, the densities of the population are increasing and decreasing periodically and the right side of this 
Fig. 1.3 implies that with the increase of time, the densities of the population are decreasing and finally is in extinction.

An example 4.4 while $k_{1}, k_{2}$ are equal and constant i.e. $k_{1}=k_{2}=4$ and $r_{1}=r_{2}=2+\exp (-t)$ are considered where $r_{1}, r_{2}$ are functions of time $t$.

Fig. 1.4 shows that both species are coexisting for uniform initial densities.

From the both left and right sides of the Fig. 1.4 with the increasing of time, the densities of the both population sizes are increasing and after a certain time the population sizes are in the equilibrium position. Here, both sketch behaviors are same since, $k_{1}, k_{2}$ are equally constant and intrinsic growth rates are same and both of them are time dependent.

In example $4.5 k_{1}, k_{2}$ are equally constant i.e. $k_{1}=k_{2}=4$ and $r_{1}=2+\exp (-t), r_{2}=9+\exp (-t)$ where $r_{1}, r_{2}$ are functions of time $t$ and $r_{1}<<r_{2}$ are considered. Then two types of solutions for two sizes of population which are represented graphically in Fig. 1.5 are obtained. In Fig. 1.5, both population sizes are coexisting for alike initial densities and with the increase of time the densities of the left sided population are increasing strictly and the densities of the right sided population are increasing and decreasing periodically for a certain time and finally the fluctuation periods tends to zero.

Example 4.3. In the following example, the intrinsic growth rates are uniform as before while $k_{2}<k_{1}$ for time dependent carrying capacities are considered.
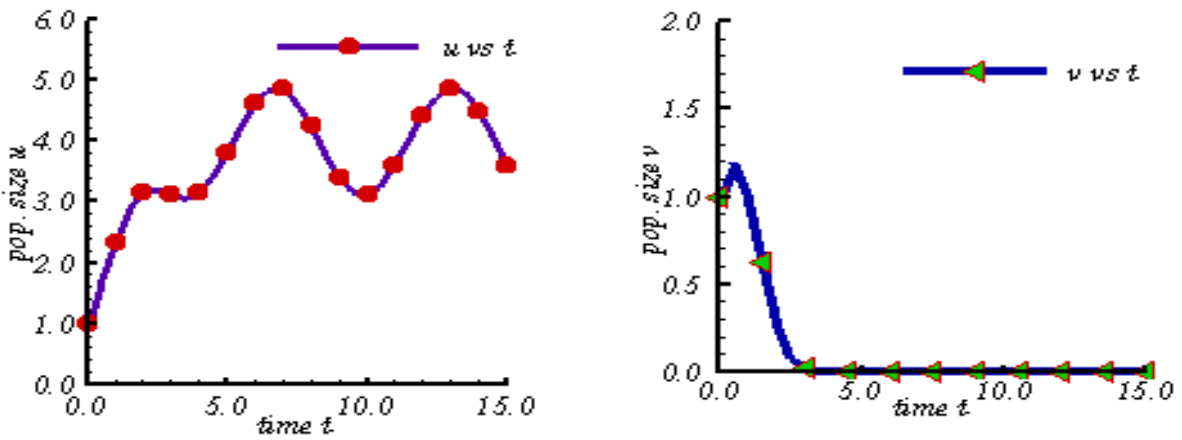

Fig. 1.3. Solutions of (9) with respect to time for $r_{1}=r_{2}=2$ and $k_{1}=4+\cos (t), k_{2}=2+\cos (t)$ and for initial values $u_{0}=v_{0}=1$.

Example 4.4. Now, we want to investigate the case for time dependent growth rates.
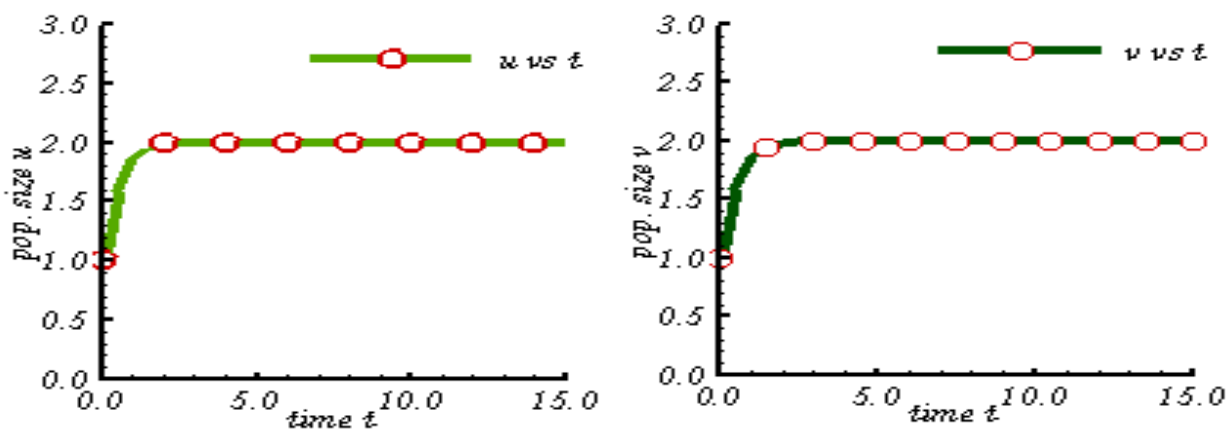

Fig. 1.4. Solutions of (9) with respect to time for $k_{1}=k_{2}=4$ and $r_{1}=r_{2}=2+\exp (-t)$ and for $u_{0}=v_{0}=1$. 
Example 4.5: Criteria when the intrinsic growth rates are unequal but time varies can be checked.
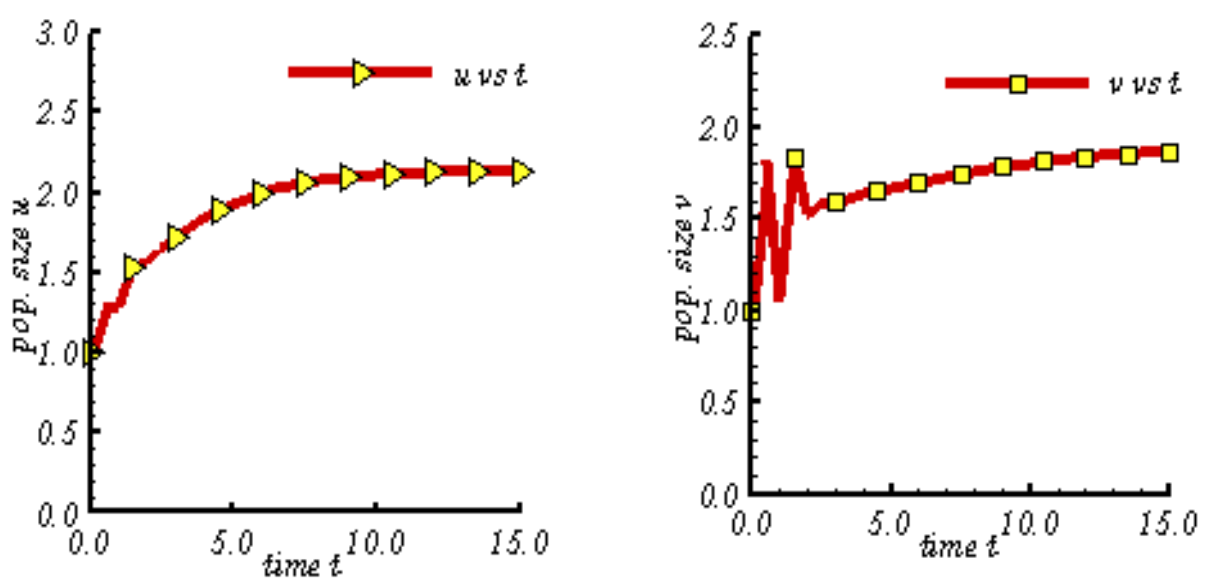

Fig. 1.5. Solutions of (9) with respect to time for $k_{1}=k_{2}=4$ and $r_{1}=2+\exp (-t), r_{2}=9+\exp (-t)$ for initial values $u_{0}=v_{0}=1$

Example 4.6. This particular example has the similar manner as designed in example 4.5 as long as $r_{2}<<r_{1}$.
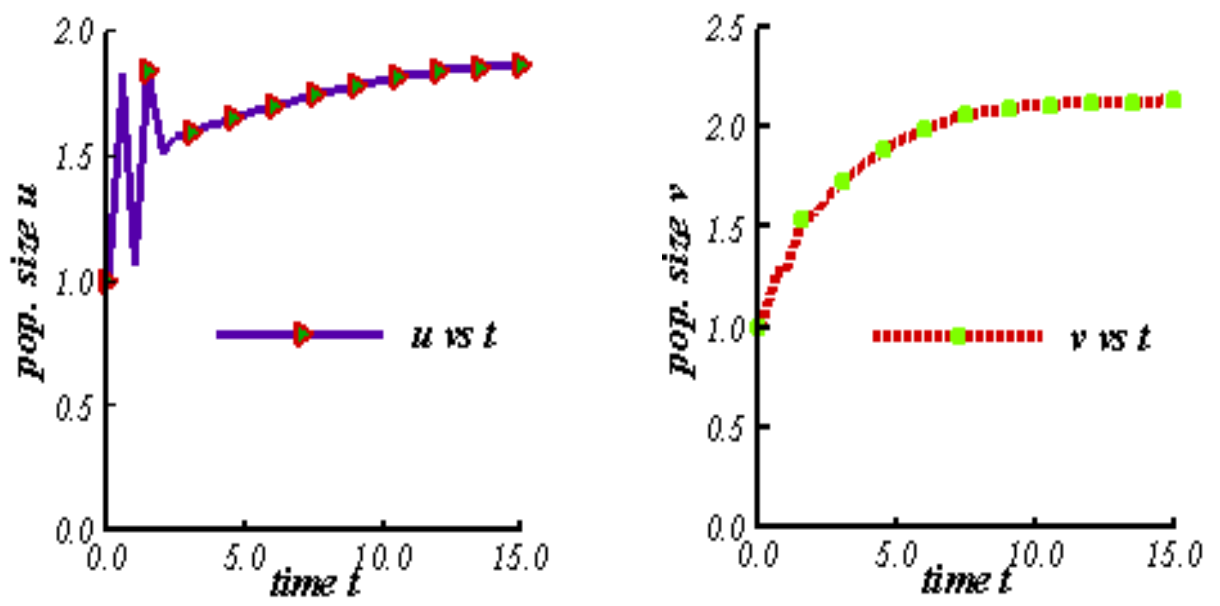

Fig. 1.6. Solutions of (9) with respect to time for $k_{1}=k_{2}=4$ and $r_{2}=9+\exp (-t), r_{1}=2+\exp (-t)$ and for initial values $u_{0}=v_{0}=1$. 
Example 4.7. The exploration for various resource functions with time variable growth rates expanding.
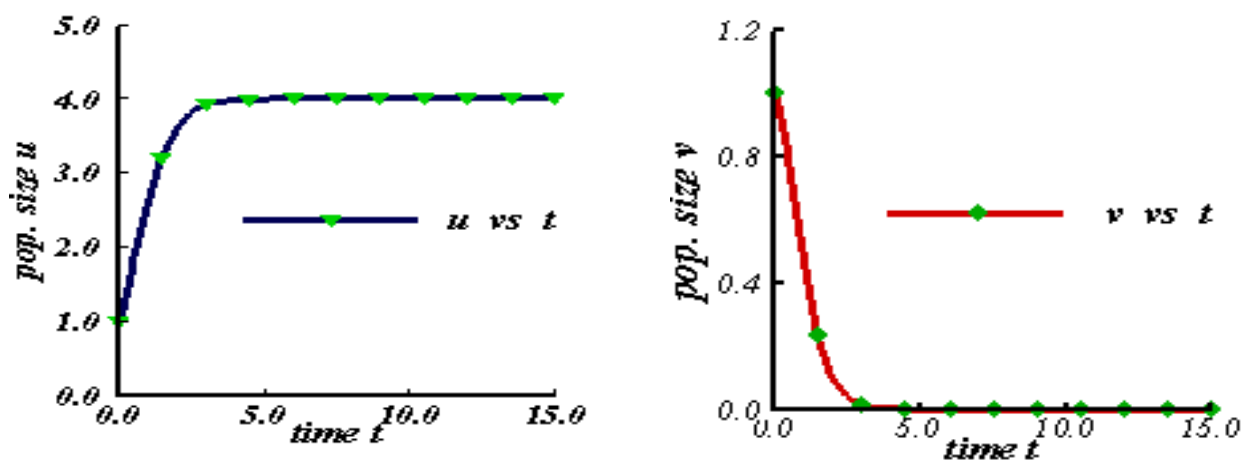

Fig. 1.7. Solutions of (9) with respect to time for $r_{1}=r_{2}=2+\exp (-t)$ and $k_{1}=4, k_{2}=2$ and for initial values $u_{0}=v_{0}=1$.

This considerable example 4.6 shows that $k_{1}, k_{2}$ are equally constant i.e. $k_{1}=k_{2}=4$ and $r_{2}=9+\exp (-t), r_{1}=2+\exp (-t)$ where $r_{1}, r_{2}$ are functions of time $t$ - In Fig. 1.6, it is clearly seen that both sizes are coexisting for similar initial densities and inverse manner as sketched in Fig. 1.5.

An example 4.7 where $k_{1}, k_{2}$ are unequal constant and $k_{1}>k_{2}$ and $r_{1}=r_{2}=2+\exp (-t)$ are considered and the results are presented graphically in Fig. 1.7. From the left design of Fig. 1.7, it is clear that the densities of the population size are increasing with the increase of time $t$ and finally after a certain time equilibrium position occurs and coincides with $k_{1}=4$ whereas the rest diagram of Fig. 1.7 implies that with the increase of time $t$ the population size are decreasing strictly and finally is in extinction.

When $k_{1}, k_{2}$ are unequal and constant and $r=r=2+\exp (-t)$ are functions of time then the results are presented graphically in Fig. 1.8 of example 4.8. In this grpah, it is seen that the left Fig. 1.8, it clearly shows that the species $u$ does not survive. From Fig.1.8, it is concluded that the solution converge to the resource function $k_{2}=4$.

Example 4.8. Considering the fact that carrying capacities are unequal and growth rates are equal but $r_{1}=r_{2} \neq$ const. and the next results are obtained.
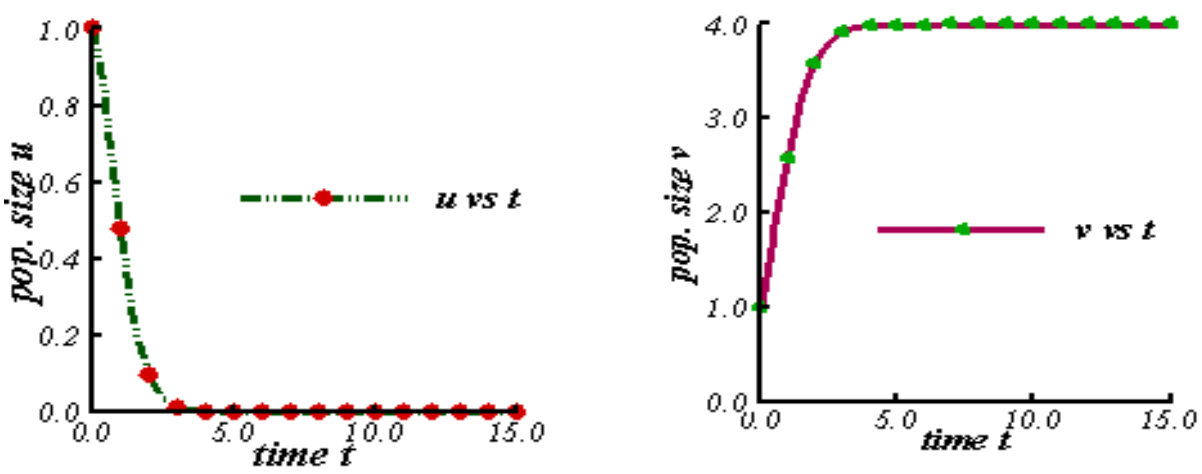

Fig. 1.8. Solutions of of (9) with respect to time for $k_{1}=2, k_{2}=4$ and $r_{1}=r_{2}=2+\exp (-t)$ and for initial values $u_{0}=v_{0}=1$. 
Example 4.9. The following study for $k_{2}>k_{1}$ whereas the growth rates are equal and nonconstant can be considered.
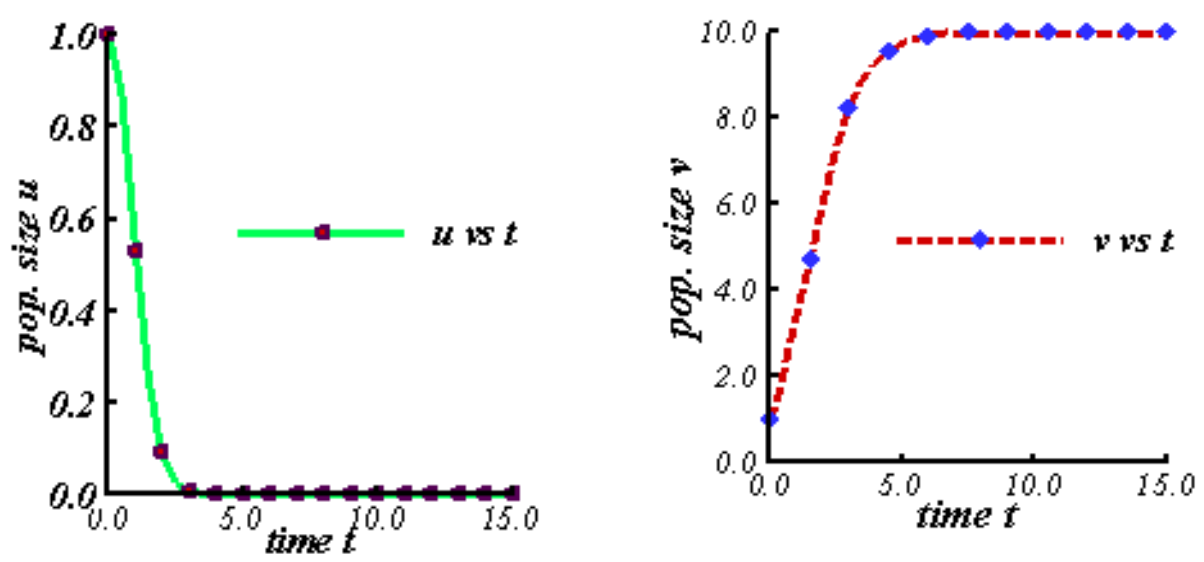

Fig. 1.9. Solutions of (9) with respect to time for $k_{1}=2, k_{2}=20$ and $r_{1}=r_{2}=1+\exp (-t)$ and for initial values $u_{0}=v_{0}=1$.

Example 4.10. Finally, all parameters are constant but unequal intrinsic growth rates can be considered.
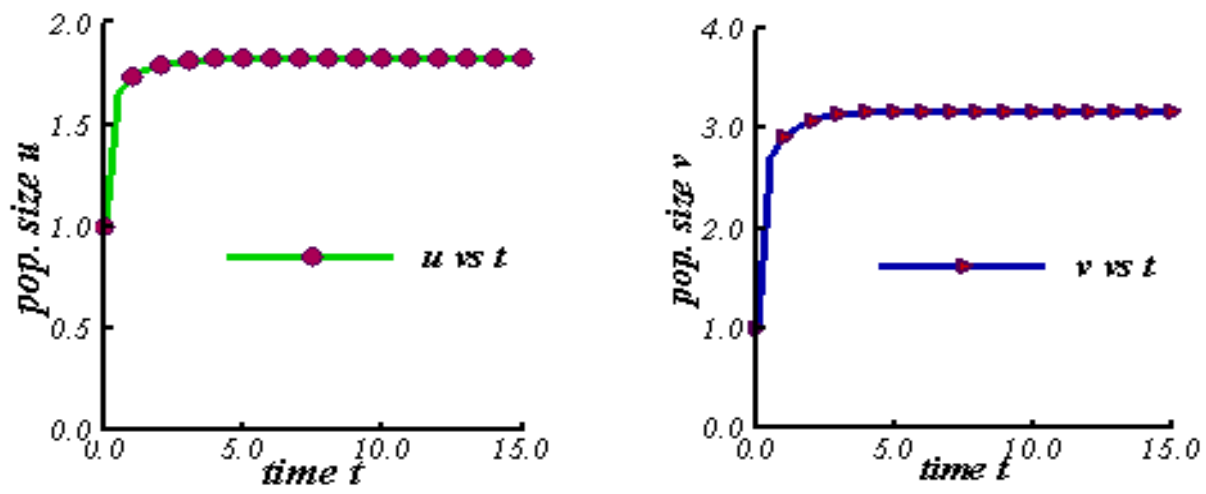

Fig. 1.10. Solutions of (9) with respect to time for $k_{1}=k_{2}=5$ and $r_{1}=3, r_{2}=6$ and for initial values $u_{0}=v_{0}=1$.

The example 4.9 is an additional one where $k_{1}, k_{2}$ are constant i.e. $k_{1}=2, k_{2}=20$ and $r_{1}=r_{2}=1+\exp (-t)$. Here $r_{1}, r_{2}$ are functions of time $t$ which are presented graphically in Fig. 1.9. The left side of this Fig. 1.9 shows that with the increase of time the densities of the population size are decreasing and tends to zero.
On the other hand, the right side of the Fig. 1.9 shows that with the increase of time, the densities of the population size are increasing strictly.

In the last example 4.10, it is considered that $k_{1}, k_{2}$ are equal and constant i.e. $k_{1}=k_{2}=5$ and $r_{1}=3, r_{2}=6$ where $r_{1}, r_{2}$ are also constant but 
not equal which are represented graphically in Fig. 1.10. In the Fig. 1.10, both organisms are coexisting for uniform initial densities. In this considerable example it is seen that with the increase of time $t$, the densities of both sizes are increasing. Lastly, it is seen that after a certain time both are in equilibrium position $(\alpha k,(1-\alpha) k)$.

\section{CONCLUSION}

The stability analyses of a non-linear system of equations have been studied and some main results established theoretically. This study is important for applications in ecology and mathematical biology. Different techniques which are used to analysis the stability of system of nonlinear differential equations have been discussed. The interested readers can use those techniques for verifying the stability of different kinds of mathematical models which are perfectly connected to our real life activities. The numerical illustrations are addressed to solve the system which is related to real world models and characterized how the parameters of each system influenced and behaviors of the solutions; consider Theorem 1 to Theorem 3 in Section 4.1. Furthermore, a very important concept is that readers can apply Lyapunov functional to verify the stability.

\section{ACKNOWLEDGEMENT}

The authors are grateful to the anonymous reviewer and Editor-in-Chief for their careful reading and suggestions. This work has been supported by University Grant Commission (UGC), Bangladesh via grant number Reg./Admin-3/65982 (Year: 2018-2019).

\section{REFERENCES}

Burden, R. L. and Faires, J. D. 1993. Numerical Analysis. Ninth Edition, PWS Publishing Company, Boston, MA.
Freedfman, H. I. 1980. Deterministic Mathematical model in population Ecology. Marcel Dekker, New York.

Gilpin, M. E. and Ayala, F. J. 1973. Global models of growth and competition, Proc. Natl. Acad. Sci. USA, 3590-3593.

Gompertz, B. 1825. On the nature of the function expressive of human mortality and on a new mode of determining the value of life contingencies. Philos. Trans. Roy. Soc.London, 513-583.

Kapur, J. N. 1989. Mathematical model in biology and medicine. Analytic Publishing Company, Delhi.

LaSalle, J. P. 1976. The stability of dynamical system. Regional Conference Series in Applied Mathematics. SIAM. Philadelphia, 1976.

Murray, J. D. 1989. Mathematical Biology. Springer, 1989.

Perko, L. 2008. Differential Equations and Dynamical Systems. Springer.

Morgan, R. 2015. Linearization and Stability Analysis of Nonlinear Problems, Hulman Undergrad. Math. J. 16(2): 1-27.

Smith, F. E. 1963. Population dynamics in Daphnia Magna and a new model for population. Ecology, 4, 651-663.

Strogatz, S. H. 2000. Nonlinear Dynamics and Chaos with Applications to Physics, Biology, Chemistry, and Engineering. Westview Press, Cambridge.

Taylor, A. 1958. Introduction to Functional Analysis. John Wiley and Sons.

Verhulst, P. F. 1838. Notice sur la loique la populations suit dans son accroissement. Correspondence Mathematique et Physique, 113-121.

Wiggins, S. 1983. Introduction to Applied Nonlinear Dynamical Systems and Chaos. Springer-Verlag, New York

(Received revised manuscript on 26 February 2019) 